\title{
Added Force-Based Methods Using Load Cells to Evaluate Walking and Tumbling with a Japanese Walking Support System
}

\author{
Shinji Kawakura*, Kiyoshi Fujiwara \\ National Institute of Advanced Industrial Science and Technology (AIST), Department of Information \\ Technology and Human Factors, Robot Innovation Research Center 1-1-1 Umezono, Tsukuba, Ibaraki \\ 305-8560 Japan. \\ * Corresponding author. Tel.: +81-80-9781-1535; email: s.kawakura@aist.go.jp \\ Manuscript submitted August 28, 2017; accepted October 28, 2017. \\ doi: 10.17706/ijbbb.2018.8.2.68-78
}

\begin{abstract}
We have conducted sequential studies to develop promising systems that address nursing challenges, and have developed applied sensing systems to solve the difficulties faced to advanced support systems for physically challenged and elderly people. In this study, our main aim was to present the utilities of the immediate and distant measurement systems by presenting qualitative and quantitative timeline data from one user of a typical Japanese walking support System. We suggested a physical analysis system using four bar-shaped metal load cells, Arduino microcomputers, and set specialized integrating and amplifying circuit boards on the arm rest to obtain data of the added weight of users on the front point of the machine. Additionally, we developed a physical analysis system, which uses two video cameras to obtain visual data of a subject simulating using programs written in Visual C++ and OpenCV packages, including the libraries and header files. Finally, we presented the multiple output values of the physical measurements obtained from various viewpoints, and examined the characters of timeline data and their kinematic parameters. We believe these mixed methodologies could be of practical use in providing alternative supports and useful directions for developers and care managers to assess and treat users' conditions.
\end{abstract}

Key words: Safe technology measurement, load cell, OpenCV, characteristic point, walking support system.

\section{Introduction}

A variety of system development techniques have been created and instituted across diverse fields including disability, rehabilitation, and sports [1]-[10]. Past scientific achievements include the execution of electric walking supporting systems (rollators); simple, non-electric systems; the tracking of characteristic points; and the drawing of stick pictures. However, limited research has examined added force on the walking supporting machine [11]-[21]. In addition, targeted achievements concerning living human bodies and non-living humanoid devices have been somewhat limited because of social and ethical issues. The aim of this study was to analyze added forces on one walking support system and visual data, using an OpenCV 2.4.9-based C++ program to calculate the parameters of the user's characteristic points when the subject used the walking support system to walk on a flat surface. Although neither human bodies nor walking support systems are simple structures, we propose using these values as references for measurements, and we thus evaluate the user's medical condition and habituation to the equipment. As there are diverse wearable electronic systems [22]-[26], we accumulated timeline data from 18 points on the subject's body 
using 3D-coordinates, acceleration, and angular velocity using a Perception Neuron (PN) motion capture system, one of the most recent powerful tools for observing physically challenged people for many years. In the future, a combination of immediate and visual methods could not only improve users' movements, but also revise nursing techniques with a combination of diverse robots [27]-[32]. Such systems could even save users from sudden or hidden diseases and injuries in outdoor and indoor settings.

\section{Theory}

\subsection{Experimental Setup}

We have conducted a sequence of studies to develop systems that address a number of nursing challenges. The experimental setup is described below. First, we categorized various walking support systems used in the fields of nursing welfare, human engineering, car production, and household electrical products based on their form, physical intensities for users, main postures, and operation (manual or electric). The various walking support systems are available for purchase worldwide, and differ according to district and lifestyle. Following preliminary research using several different systems, we considered shape, weight, size, and above all, prevalence to select one typical Japanese walking support system, the Rollator KA-391 (Paramount Bed Co., Ltd., Japan), for use in this study. The unit is $660 \mathrm{~mm}$ wide, $732 \mathrm{~mm}$ long, adjustable between 966-1,218 $\mathrm{mm}$ high, and weighs $12.5 \mathrm{~kg}$. The shafts are made from aluminum, and the points where subjects put their elbows (on the elbow rest) are made of plastic. Subjects can grasp the handle anywhere with their non-dominant hand. Second, we examined the actions of 1) walking straight for several hundred meters at usual speed and 2) falling in four directions (forward, backward, to the right, and to the left), because these are common full-body movements. When simulating a fall, to prevent his body from suddenly falling away from the machine, the user was asked to grasp the handle with bar-shaped load cells (Weight Sensor (Load Cell) 0-50kg 114990100 (Seeed Studio Inc., U.S.)). These load cells are placed such that he could grasp them while resting his elbow bone on the other load cells. The load cells were connected to Arduino microcomputers (Arduino UNO (Arduino Inc., Italy)) and specialized integrating and amplifying circuit boards (HX711l load cell amplifier SEN-13879 (Spark Fun Inc., U.S.)) with common electrical connectors and lead lines connected to two small, light laptop PCs for gathering data in real time. We fixed one of the PCs against the main aluminum shaft of the walking support machine with wide, tough bands, and the other in the knapsack the subject wore on his back. We fixed the load cells at points (1) and (2) in Fig. 1-2, such that his elbows would rest on the two load cells (3) and (4) in Fig. 1-2. Additionally, the subject wore a peripheral, default harness, which attached him to the machine at the waist and hips to keep his body from suddenly falling away from the machine (Fig. 3).

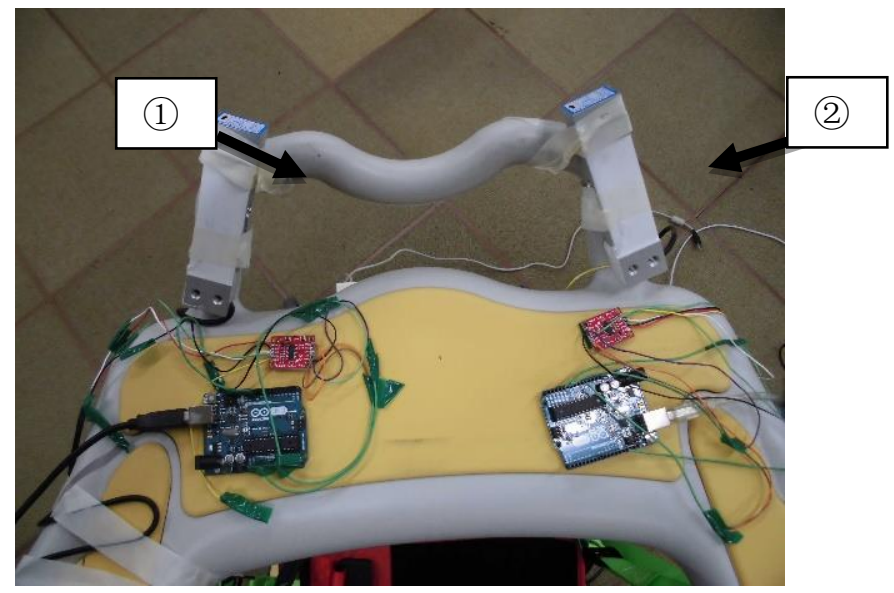

Fig. 1. Walking support system and sensing systems. 


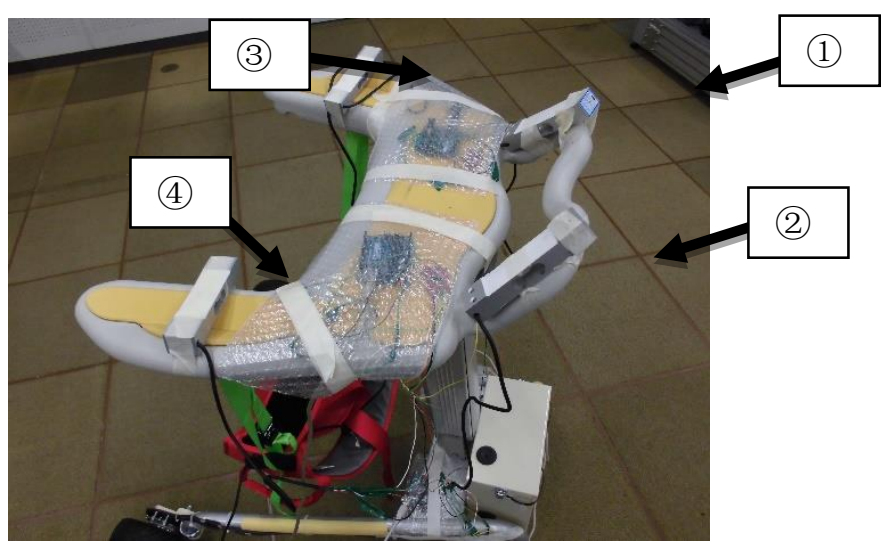

Fig. 2. Walking support system and sensing systems.

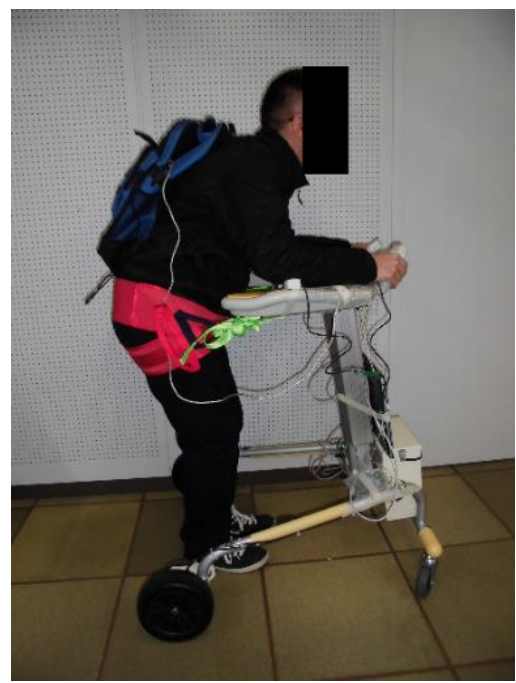

Fig. 3. Walking support system and subject.

The user executed the aforementioned movements five times successively, with few-minutes intervals between each trial. However, it was difficult to know whether the characteristics and number of tasks, the experiment sets, time interval, and situation were appropriate. We selected one Japanese male subject with the appropriate body size and features. He was $172 \mathrm{~cm}$ tall and weighed $63 \mathrm{~kg}$; the average Japanese elderly (60-64 years old) male is $167.8 \mathrm{~cm}$ tall and weighs $66.0 \mathrm{~kg}$ (Ministry of Health Labor and Welfare, Japan, 2015) , and we judged that those values were close. Our subject was 38 years old at the time of the experiment. One of our main aims was to verify our systems and obtain physical data. According to a preliminary interview, the subject fulfilled the criteria of no serious diseases, peculiar habits, or prior relevant careers, such as being an expert in sports or martial arts. To collect fundamental information about the subject, we used a questionnaire with previously established reliability from the Japan Association of Industrial Health and other health organizations. Furthermore, we checked the subject's level of fatigue after each trial, and we evaluated his fitness level, smoking habits, and the occurrence of backache to establish his physiological and medical condition. In line with similar studies in which researchers have measured the motion and position of the subject's bones, but not the motion and position of his muscles. For the camera system setup, we selected two common, nonspecialized, medium-format digital video cameras of model HDR-PJ790V (SONY, Japan). We chose these more universal cameras rather than the specialized high-speed cameras used in the fields of human dynamics and sports sciences in consideration of the potential future uses of such measurement systems. The camera sampling rate was $30 \mathrm{fps}$ (frames per 
second), and the frame size was $1280 \times 720$ pixels (16:9), with standard mode settings. The distances from the fixed cameras to the subject, when the subject was static (not walking), were 3.0 meters from the side and 1.5 meters from the front (Fig. 4). After obtaining the visual data, we converted the files into AVI format. The frame size and frames per second (fps) of the visual data were uniformly converted to $320 \times 240$ pixels. This conversion was done to ensure compatibility with a Visual C++ based program and the OpenCV library and header files. Based on the findings of past studies, these settings are reasonable for use in the analyses conducted by general nursing wor kers and other social scientists, directors, and managers to obtain a time series of visual data for diverse analyses of indoor experiments. Although many existing studies use the visual data capturing library, header files, or program codes from OpenCV ver. 2.4.9 to obtain visual data, we lacked confidence in these methods because of their potential negative impact on recording accuracy. We thus used these elements only for the analyses, after the visual data were processed. This immediate load-cell based system and the non-immediate measurement methodology were low-cost and easy to set up compared to similar previously implemented systems.

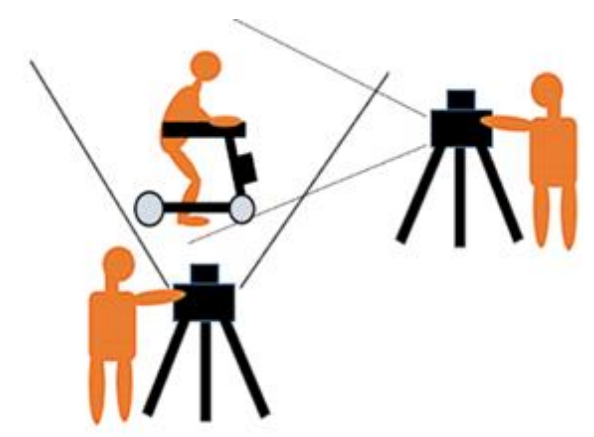

Fig. 4. Camera, walking support system, and subject set-up.

We conducted our experiments on a flat surface at the National Institute of Science and Technology (AIST) and consolidated the other experimental field settings as much as possible. We also ensured that the subject's clothes were appropriate for the physical demands of the experiment and for the visual data analysis. The subject wore a black jumper, casual pants, and shoes, similar to the casual, generic fashions of Japan. The clothing was thin and tight to minimize the chances of the sensor nodes sliding. The background walls and floor of the space were a neutral (not tinted) white color, and one white door was located on the right side of the wall. The white soles of the shoes did not substantially affect the visual analysis. We confirmed the adequacy of the color contrast using computer program-based processes. As the clothing contained no protruding items or accessories, it was appropriate for the experiments. We conducted visual analyses by calculating the differences between two successive frames of the visual data..

\subsection{Data Analysis Methods}

We analyzed the visual data using original programs written in Visual $\mathrm{C}++$, including the OpenCV library and header files. This section explains the theories underlying our image analyses. We used Shi-Tomasi's method and the function cvGoodFeaturesToTrack in the OpenCV library to execute an automatic search for the characteristic points in selected pictures. Although the Harris method can be used to change parameters, we did not use it in this study for reasons discussed below. The Shi-Tomasi and Harris methods are mathematically similar. When focusing on the characteristic points that are easy to distinguish from nearby pixels and slightly shifting the targeted picture in the analysis area, it is possible to detect and record the points where the sum of the squared difference $(S S D)$ becomes larger than at other points. The SSD is defined as shown in (1) and (2), when shifting the picture by a sufficiently small $\Delta v$ in the window $W$, where the center is point $p$. 


$$
\begin{gathered}
S S D=S_{(p)}=\sum_{\mathrm{q} \in \mathrm{W}}^{n}(I(q)-I(q+\Delta v))^{2} \\
S(p)=X q \in W(I(q)-I(q+\Delta v))^{2}
\end{gathered}
$$

Furthermore, to execute a Taylor development and primary approximation about the pixels of the shifted picture, $I(q+\Delta v)$ can be expressed as shown in (3), using $I(q)$ 's partial differentials with respect to $x$ and $y$ $(I x(q)$ and $I y(q)$, respectively).

$$
I(q+\Delta v) \fallingdotseq I(q)+\left[I_{x}(q) \cdot I_{y}(q)\right] \Delta v
$$

These two equations can be combined so that $S(p)$ is expressed as shown in (4).

$$
S_{(p)}=\Delta v^{\mathrm{T}}\left[\begin{array}{cc}
\sum_{W} I_{x}^{2} & \sum_{W} I_{x} I_{y} \\
\sum_{W} I_{x} I_{y} & \sum_{W} I_{y}^{2}
\end{array}\right] \Delta v=\Delta v^{\mathrm{T}} H_{(p)} \Delta v
$$

The matrix $H(p)$ presents the features of the luminance distribution around point $p$. In the theories of Shi-Tomasi, the feature value $M$ from the characteristic value of matrix in (4) is defined as in (5), and is used for detecting the feature points.

$$
M=\min \left(\lambda_{1}, \lambda_{2}\right)
$$

By contrast, using the theory of Harris, we define the characteristic value of the matrix as in (6), as $\lambda_{1}$ and $\lambda_{2}$, with $\kappa$ as a parameter. When both $\lambda_{1}$ and $\lambda_{2}$ are sufficiently large, the appropriate feature points are defined as one. Then, the Harris operator can detect the specific points that make the value of $M$ in (6) sufficiently large.

$$
M=\lambda_{1} \cdot \lambda_{2}-\kappa\left(\lambda_{1}+\lambda_{2}\right)^{2}=\operatorname{det}(H)-\kappa \cdot \operatorname{trace}^{2}(H)
$$

We used the Shi-Tomasi method in this study because corner detection is related to its precision and burden (time and effort), whereas, with the Harris operator, the program simply judges the corners from the balance between the value of M and threshold $\kappa$. In almost all cases, the value of $\kappa$ must be obtained by performing a sufficient number of experiments. We used the Kanade method and optical flow to execute automatic and manual searching for characteristic points in selected pictures. These are powerful and well-established methods in this scientific field. The outline of the procedure is as follows: 1) Determine the optical flow vectors using the least squares method, 2) identify the differences between the real values and the estimated solutions, and 3) use the estimated solutions to the real values using the hill climbing method. The flow of the Kanade method involves creating a smoothing filter to cover (or wrap) pictures $H$ and $I$, pruning the pixels, and making the new pictures $H_{1}$ and $I_{1}$, such that the resolution is halved (the number of pixels is quartered and the pixel size is doubled). The procedure is repeated to make $H_{2}, I_{2}, H_{3}, I_{3} \ldots H_{\mathrm{n}}, I_{\mathrm{n}}$. The "pyramids of pictures" are developed based on $H$ and $I$ until the pixel size in these pictures becomes larger than the maximum "travel distance". As was the case in prior processing, the approximate solutions of travel distance are set to $u=0$ and $v=0$, and the rank of picture, $i$ is set to $n$. We calculate each value based on the aforementioned methods as a loop of processions. Next, we calculate the value of $\Delta u$ and $\Delta v$ for $H_{i}$ and $I_{i}$, the values of the travel distance $\left((u, v)=\left(u+\Delta u, v^{\prime}+\Delta v\right)\right)$ for each pixel, and the approximate values of travel distance $\left(u,{ }^{\prime} v\right)$ for the picture one rank higher $\left(H_{i}-1, I_{i}-1\right)$ about the resolution using the methods below. The pixel-points $(2 u, 2 v)$ are set (this picture has $(x / 2 \times y / 2)$ pixels, where $x$ and $y$ are both 
odd numbers), and the approximate travel distance is $I=\left(u^{\prime}, v^{\prime}\right)$. Based on the other pixels, we define the average values of travel as $\left(u^{\prime}, v^{\prime}\right)$ for only the odd-numbered pixels along the four sides (upper, lower, right, and left sides). In short, it is a twin linear complement. Next, we subtract one from the current $i$ number (in the program code, $i=i-1$ ) and repeat the procedure until the value of $i$ reaches zero. We set the values of $u$ and $v$ based on $u^{\prime}=2 u$ and $v^{\prime}=2 v$, and the values of $\Delta u$ and $\Delta v$ based on $u^{\prime \prime}=2\left(u^{\prime}+2 \Delta u\right)$ and $v^{\prime \prime}=2\left(v^{\prime}+2 \Delta v\right)$, and we repeat the sequences from $i=n$ to $i=0$ in the program, while decreasing the value of $i$ by one in each iteration. In Step 4), using the theory of motion template, we successively update the motion history image and process the obtained visual data for various motion analyses and object tracking tasks using a "moving silhouette" contained in OpenCV. First, we used a function named updateMotionHistory from the C++ version. By estimating and indicating following arguments [1]-[4], these functions can be handled flexibly using the following arguments: 1) silhouette-a silhouette mask that has non-zero pixels where the motion occurs; 2) $m h i$-a motion history image, which is updated by the function (single-channel, 32-bit floating-point); 3) timestamp - the current time in milliseconds or other units; and 4) duration-the maximal duration of the tracked motion in the same units as timestamp. The function updates the motion history image as follows:

$$
\operatorname{mhi}(x, y)= \begin{cases}\text { timestamp } & \text { if } \operatorname{silhouette}(x, y) \neq 0 \\ 0 & \text { if } \operatorname{silhouette}(x, y)=0 \text { and mhi }<\text { (timestamp }- \text { duration }) \\ \operatorname{mhi}(x, y) & \text { otherwise }\end{cases}
$$

Equation (7) shows that the mhi pixels where motion occurs are set to the current timestamp, whereas the pixels where the motion last happened a long time ago are cleared. The function, with two additional important functions-calcMotionGradient and calcGlobalOrientation-implements a motion template technique. We selected and executed a measurement and observed the added forces on load cells on the machine, and then automatically set and located the characteristic points. Next, we analyzed each timeline's numerical data using significant statistical methodologies. Using a common, mid-range, digital camera and the latest OpenCV libraries, we obtained visual data and formatted them for the next procedure. Then, we used our original program to automatically set 24 characteristic points, followed the location of the characteristic points through time, and analyzed later at the later time.

\section{Results}

The numerical results of this study were analyzed and discussed based on the findings of previous studies. To assess the subject's movements when he was walking, and to demonstrate the methodological utilities, we calculated and output the averaged timeline data of five trials for each indicator. To examine the subject when falling, we calculated the average data after lining up the peak points of the graphs. Figs. 5 show the averaged timeline data from four load cells [33]. Figs. 6 show bar charts showing the contrast in the values of added forces on four load cells. Fig. 7 shows one example of the visual data obtained from the cameras and the 24 characteristic points automatically drawn by the camera. However, realistically speaking, subtle time-delays typically occur in the drawing speed of the characteristic points in the small window of the Visual C++ environment. Nevertheless, when computing the characteristic points, a delay was not observed. Fig. 8 shows the average timeline coordinate data (about the $\mathrm{x}$ and $\mathrm{y}$ axis) of the 24 characteristic points from visual data analysis. We suppose that these findings indicate that the systems used are appropriate for our Visual C++ environment for AVI styled video data. Another program was then used to indicate, mark, and erase the characteristic points both automatically and manually (by clicking on them with the mouse). After marking 24 points automatically, we traced them. The features and accuracy of the set of characteristic points were input automatically, and we accumulated sequential timeline data for the $\mathrm{x}$ - and 
$y$ - coordinates of each point in two dimensions. Those timeline data appeared to indicate the subject's dynamism and their motion characteristics. However, other elements (e.g., biomechanical forces) showed the characteristics of the subject's movements, according to their physical attributes.

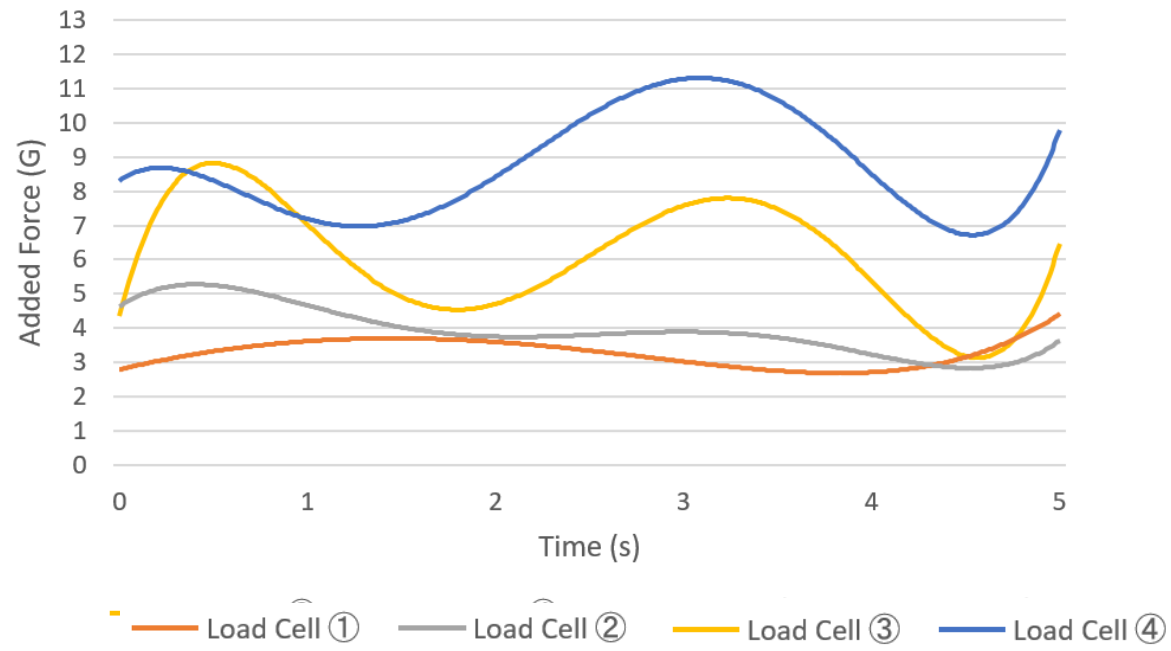

Fig. 5. Timeline chart (spline interpolation was applied) of the added force on the load cells for the walking subject.

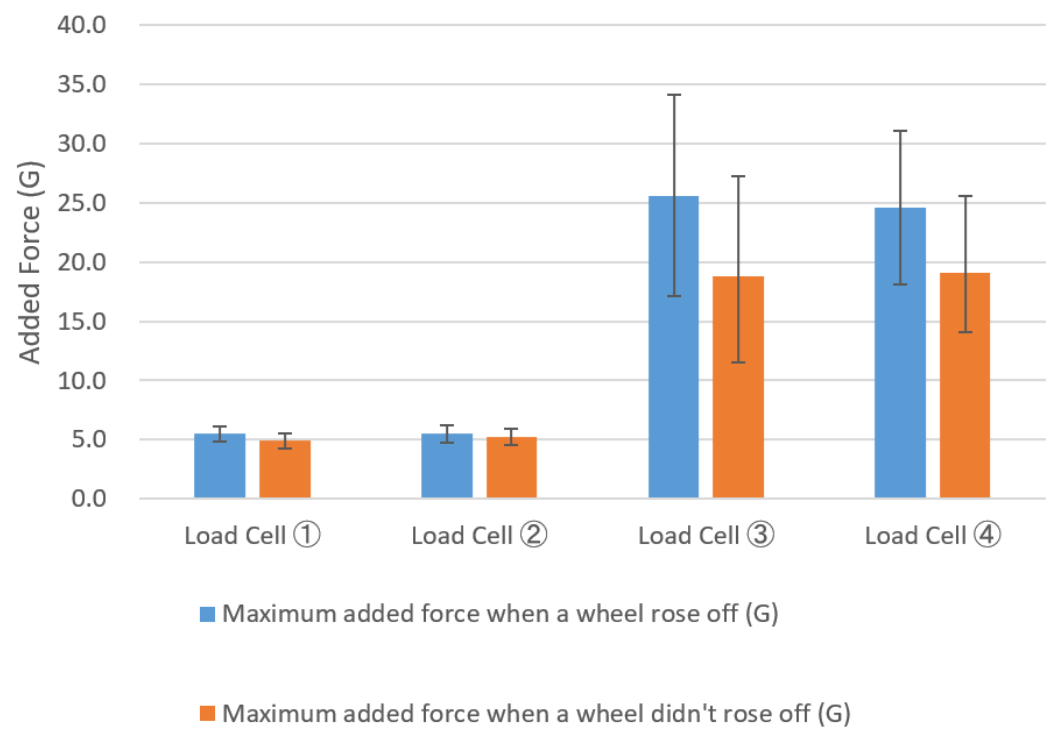

Fig. 6. Bar chart showing the contrast in the values of added forces on four load cells.

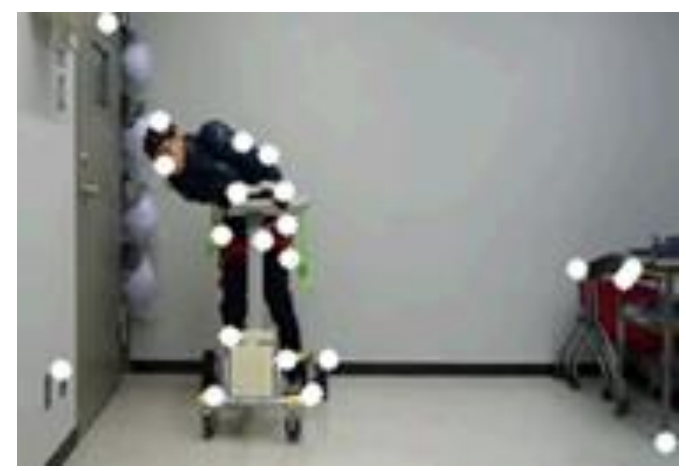

Fig. 7. Twenty-four characteristic points drawn automatically on the visual data. 


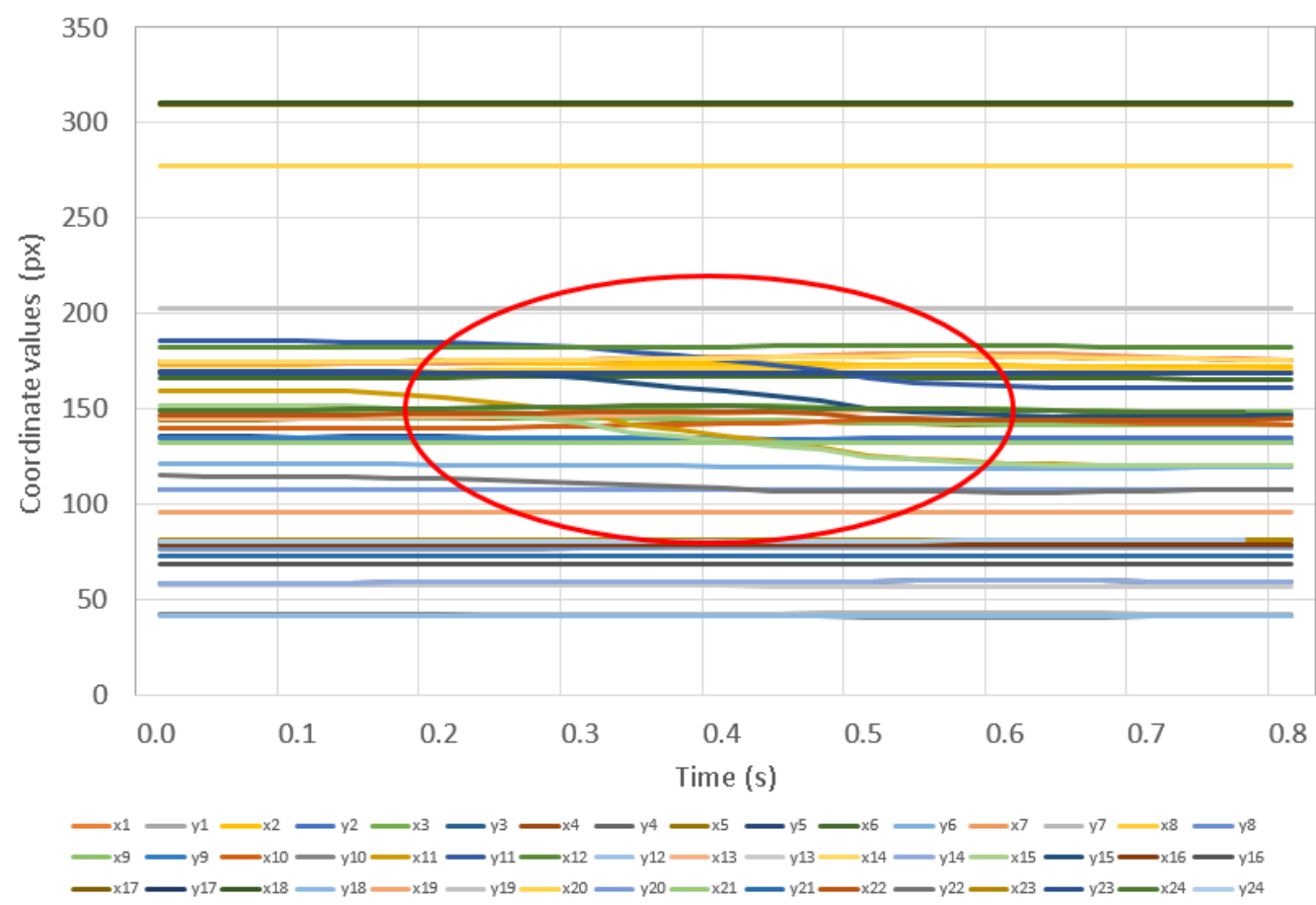

Fig. 8. The average timeline data of the coordinates of 24 automatically-set characteristic points on the subject while he was falling to his right, captured by the front camera.

\section{Discussion}

As mentioned above, Fig. 5 shows the added forces on four load cells to be smoothly curving lines. Those data are in the common range for this industrial field. However, for the data presented in Fig. 5, there was not enough previous concrete data because of the facilities, shape, and lightness of the walking supporting system, so we had difficulty commenting on those data. In Fig. 6, we contrasted the added force data from trials where one of four wheels rose off the floor and those where it did not based on the observation of the experiment operator. Fig. 8 shows the 24 automatically-set characteristic points on the subject's video when he was falling to his right and left sides, captured by the front cameras. We found clear evidence of the raw data curves changing in relation to the previously defined load cells' data and automatically-set characteristic points. However, it is difficult to interpret those numerical features because of the methodological limitations and the complex forms of the curves. Nevertheless, we consider that the proposed sensing methodologies, viewpoints, and concepts could be used in practical caregiving and physical training fields. The data sets shown in Figs. 5-8 could be used to check the stability of the walking support system and the suitability of the system's settings concerning, for instance, weight-balance, size, and the user's muscles. As seen in Fig. 8, we were able to measure the changes in the 24 curves when the subject was falling. This seems to be a meaningful accumulation of data from the PN system.

\section{Conclusion}

In this study, we proposed measurement and analysis methods for use with physically challenged and elderly people, using a physical analysis system of four bar-shaped metal load cells, Arduino microcomputers, and circuit boards, including amplifiers, integrated into the arm rest of a walking support system. By obtaining data of added weight on the front point of the walking machine and characteristic values of distant visual data from OpenCV-based program, we estimated those systems' utilities and various physical indicators were promising for future prospects. We also confirmed and reconsidered various measurement and performance limitations of these extremely low-cost systems. The noteworthy point is 
that, even with these limitations, we obtained meaningful results and the consequent benefits, specifically related to users' falling, from the study. In future, at research institutes, academic institutions, retirement homes, and hospitals, those results could assist researchers, physically handicapped persons, and care workers to overcome difficulties in grasping subtle changes and obtaining comprehensive conditions of human bodies and systems. Furthermore, future studies will determine those systems' validity, durability, precision, and social impact. Ideally, diverse subsequent studies should aim to obtain a greater variety of data from a larger number of subjects, and focus on additional forces targeting other ages, races, and movements to facilitate comparisons with our data in this study, considering not only the supreme complexity of the human body's structure and differences from individual to individual, but also a diversity of analysis methodologies, specific experimental conditions and spaces.

\section{Acknowledgment}

Our heartfelt appreciation goes to colleagues at the National Institute of Advanced Industrial Science and Technology (AIST), members of Impulsing Paradigm Change through Disruptive Technologies (ImPACT) Program/Cabinet Office, Government of Japan, for their considerable support, feedback, and valuable comments.

\section{References}

[1] Augustyniak, P., Smoleń, M., Mikrut, Z., \& Kańtoch, E. (2014). Seamless tracing of human behaviour using complementary. Sensors, 14(5), 7831-7856.

[2] Fang, J., Xie, Q., Yang, G. Y., \& Xie, L. (2017). Development and feasibility assessment of a rotational orthosis for walking with arm swing. Frontiers in Neuroscience, 11(32), 1-10.

[3] Huang, J., Xu, W., Mohammed, S., \& Shu, Z. (2015). Posture estimation and human support using wearable sensors and walking-aid robot. Robotics and Autonomous Systems, 73, 24-43.

[4] Karantonis, D., Narayanan, M., Mathie, N. L., \& Celler, B. (2006). Implementation of a real-time human movement classifier using a triaxial accelerometer for ambulatory monitoring. Information Technology in Biomedicine, IEEE Transactions, 10(1), 156-167.

[5] Tanaka, E., Muramatsu, K., Watanuki, K., \& Yuge, L. (2015). Walking assistance apparatus enabled for neuro-rehabilitation of patients and its effective-ness. Mechanical Engineering Letters, 1(0), 15-00530.

[6] Masiero, S., Poli, P., Rosati, G., \& Zanotto, D. (2014). The value of robotic systems in stroke rehabilitation. Expert Rev. Med. Devices Early online, 1-12.

[7] Mukhopadhyay, S. C. (2015). Wearable sensors for human activity monitoring. A Review: IEEE Sensors Journal, 15(3), 1321-1330.

[8] Raveendranathan, N., Galzarano, S., Loseu, V., Gravina, R., Giannantonio, M., Sgroi, J. R., \& Fortino, G. (2012). From modeling to implementation of virtual sensors in body sensor networks. IEEE Sensors Journal, 12(3), 583-593.

[9] Suzuki, S., Hirata, Y., Kosuge, H., \& Onodera, H. (2013). Motion support during the swing phase using cooperative walking support system. Advanced Robotics, 27(17), 1337-1349.

[10] Zhou, F., Torre, F., \& Hodgins, J. K. (2013). Hierarchical aligned cluster analysis for temporal clustering of human motion. IEEE Trans. on Pattern Analysis and Machine Intelligence, 35(3), 582-596.

[11] Chen, Z. (2009). Efficient block matching algorithm for motion estimation. International Journal of Signal Processing, 5(2), 133-137.

[12] Ohnuma, T., Lee, G., \& Chong, N. Y., (2014). Particle filter based lower limb prediction and motion control for JAIST active robotic walker. Proceedings of the 23rd IEEE International. Symposium on Robot and Human Interactive Communication (pp. 6-11). 
[13] Kabalan, C., \& Badr, G. (2016). Computer vision guidance system for indoor navigation of visually impaired people. Proceeding of 2016 IEEE 8th International Conference on Intelligent Systems (IS), 449-454.

[14] Valls, M. J., Poon, J., \& Huang, S. (2012). Low-cost visual tracking with an intelligent wheelchair for innovative assistive care. Control Automation Robotics \& Vision (ICARCV), 1540-1545.

[15] Dulari, S. (2016). Automatic camera based eye controlled wheelchair system using raspberry pi. International Journal of Science, Engineering and Technology Research (IJSETR), 5(1).

[16] Pradhan, P. P., \& Dhanure, P. S. (2015). Child activity monitoring using tri-axial accelerometer. International Journal of Advanced Research, 3(6), 222-224.

[17] Hossain, E., Khan, M. R., Muhida, R., \& Ali, A. (2013). Analysis and implementation for a walking support system for visually impaired people. International Journal of Intelligent Mechatronics and Robotics archive, 1(3), 46-62.

[18] Jiang, Y., Wang, S., Ishida, K., \& Kobayashi, Y. (2014). Directional control of an omnidirectional walking support walker: Adaptation to individual differences with fuzzy learning. Advanced Robotics, 28(7), 479-485.

[19] Dine, K. M. E., Yang, C., Nozaki, T., \& Murakami, T. (2016). Novel walking assist device based on generic human motion tracking criteria. Proceedings of 2016 IEEE 14th International Workshop on Advanced Motion Control (AMC) (pp. 38-43).

[20] Ko, C., Young, K., Huang, Y., \& Agrawal, S. K. (2013). Active and passive control of walk-assist robot for outdoor guidance. IEEE/ASME Transactions on Mechatronics, 18(3), 1211-1220.

[21] Ko, C., Young, K., Huang, Y., \& Agrawal, S. K. (2013). Walk-assist robot: A novel approach to gain selection of a braking controller using differential flatness. IEEE Transactions on Control Systems Technology, 21(6), 2299-2305.

[22] Pritchard, E. D., Lee, Y., \& Bao, L. (2008). Mathematical learning models that depend on prior knowledge and instructional strategies. The American Physical Society (Physical Review Special Topics Physics Education Research), 4(1), 010109-1-010109-8.

[23] Qiu, S., Wang, Z., Zhao, H., \& Hu, H. (2016). Using distributed wearable sensors to measure and evaluate human lower limb motions. IEEE Transactions on In-strumentation and Measurement, 65(4), 939-950.

[24] Satoshi, M., \& Kaori, F. (2011). Stabilization of projected image for wearable walking support system using pico-projector. IEEE 17th International Conference on Embedded and Real-Time Computing Systems and Applications (RTCSA), 113-116.

[25] Mukhopadhyay, S. C. (2015). Wearable sensors for human activity monitoring. A Review: IEEE Sensors Journal, 15(3), 1321-1330.

[26] Kawakura, S., \& Shibasaki, R. (2013). Supporting systems for agricultural worker's skill and security. Proceedings of Asian Conference Remote Sensing (ACRS) (pp. 71-77).

[27] Machida, E., et al. (2012). Human motion tracking of mobile robot with Kinect 3D sensor. Proceedings of IEEE SICE Annual Conference 2012 (pp. 2207-2211).

[28] Wei, Y., Xiangxin, K., \& Lee, M. C. (2014). A new vision and navigation research for a guide-dog robot system in urban system. Proceeding of IEEE/ASME International Conference on Advanced Intelligent Mechatronics (AIM) 2014.

[29] Said, A. M., et al. (2013). Real-time vision based dynamic linkage detection for exploration rovers. 2013 IEEE/RSJ International Conference on Intelligent Robots and Systems (IROS).

[30] Kuo-Lan, S., Li, B. Y., \& Kuo-Hsien, H. (2015). Development of Tracking System for Mobile Robot Using Image Recognition Algorithms. Transactions of the Canadian Society for Mechanical Engineering, 39(3), 501-513. 
[31] Mehta, S. S., \& Burks, T. F. (2014). Vision-based control of robotic manipulator for citrus harvesting. Computers and Electronics in Agriculture, 102, 146-158.

[32] Fontanelli, D., Giannitrapani, A., \& Palopoli, L. (2015). A passive guidance system for a robotic walking assistance using brakes. Proceedings of 2015 IEEE 54th Annual Conference on Decision and Control (CDC), 829-834.

[33] Murao, T., Hirao, Y., \& Hashimoto, H. (2011). Skill level evaluation for taijiquan based on curve fitting and logarithmic distribution diagram of curvature. SICE Journal of Control, Measurement, and System Integration, 4(1), 1-5.

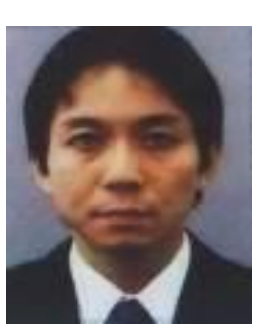

Shinji Kawakura was born on July 14, 1978. He is research associate at National Institute of Advanced Industrial Science and Technology (AIST), Department of Information Technology and Human Factors. He received the Ph.D. in environmentology, the University of Tokyo, 2015.

He got the B.A in control system engineering, Tokyo Institute of Technology, 2003; the M.A. in human-factors engineering, Tokyo Institute of Technology, 2005.

He worked as system engineering, researching for private companies, development and verification of wearable sensing systems with real-time spoken commands for outdoor agricultural workers.

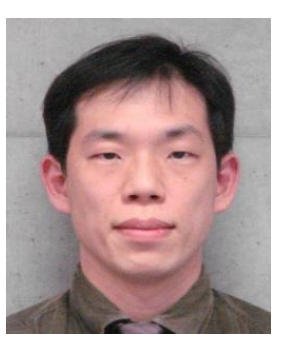

Kiyoshi Fujiwara was born on February 22, 1972. He is senior researcher at National Institute of Advanced Industrial Science and Technology (AIST), the Department of Information Technology and Human Factors. He received the Ph.D. in engineering, Tsukuba University. 